\title{
THE PRINCETON EXPERIMENT ON THE ORBITING ASTRONOMICAL OBSERVATORY
}

\author{
E. B. JENKINS \\ Princeton University Observatory, Princeton, N.J., U.S.A.
}

Princeton's $80 \mathrm{~cm}$ diameter telescope with an ultraviolet spectrometer will be the prime experiment on board NASA's third Orbiting Astronomical Observatory (OAO-C) which is scheduled for launch in late 1971. Two scanning carriage arms within the spectrometer will each have one photomultiplier sensitive to a 1600-3200 first order spectrum and another photomultiplier which will register from 800 to $1600 \AA$ in the second order. Since the primary purpose of the instrument will be to study interstellar absorption lines, the design philosophy has emphasized the role of carefully examining the detail of specific lines, rather than surveying a star's entire spectrum. Thus the carriage with the narrowest exit slits, providing $0.1 \AA$ resolution in first order and $0.05 \AA$ in second order, has been programmed to repetitively scan and retrace over any selected $0.7 \AA$ interval in first order (or $0.35 \AA$ in second order). Stepping motion in one direction every $16 \mathrm{~s}$ will occur for the second carriage, which will offer 0.4 and $0.2 \AA$ resolutions for first and second orders, respectively. Approximately half of the starlight which is focussed on the entrance slit jaws is reflected into a fine guidance system which will enable the spacecraft to stabilize to within 0.1 arc sec on a star as faint as 7 th magnitude.

As an example of the overall photon detection efficiency of the instrument, a moderately reddened 5 th magnitude $\mathrm{B} 0$ star would produce 40 counts $^{-1}$ within a $0.05 \AA$ passband near $1400 \AA$. Hence in one orbit a signal-to-noise ratio of 100 could be attained over $0.7 \AA$ and it follows that one could conceivably extract roughly 5000 times this much information from 5 th magnitude stars during the anticipated one year lifetime of the OAO. Much of the light from any extended object will be rejected by the entrance slit whose width subtends only 0.3 arc sec in the sky. A four times wider slit may be selected for observations at a reduced resolution, and one should, for instance, be able to obtain about 16 counts s $^{-1}$ at $0.4 \AA$ resolution near $2000 \AA$ for Mars or Jupiter at opposition.

\section{DISCUSSION}

J.-L. Bertaux: Do you have some aging problems with the photocathodes of open photomultipliers?

$E$. B. Jenkins: Yes. One must be careful to store the open photomultipliers in a low humidity environment.

$W$. Werner: What did you do for the protection from stray light by the edges of the entrance slit?

$E$. B. Jenkins: The entrance slit is not directly visible to the data photomultipliers, and any light scattered off the slit edges would probably have to scatter several times within the spectrometer before it would have any probability of entering a photomultiplier.

R.J. Van Duinen: 1. What does L i sky emission do to your shortest wavelength observations?

2. What is the calibration source like? 
E. B. Jenkins: 1. Diffuse emission should have an almost negligible effect on our instrument since the entrance slit on the spectrometer accepts a very small solid angle.

2. The calibration source allows us to check the relationship of wavelength versus carriage arm position while the spacecraft is in orbit. On command from the ground, one of two iron-neon discharge sources illuminates a small mirror next to the entrance slit inside the spectrometer. 